\title{
Autoradiography-based, Three-dimensional Calculation of Dose Rate for Murine, Human-tumor Xenografts
}

\author{
KENNETH F. KORAL ${ }^{*}$, CHEUK S. KWOK ${ }^{2}$, FARLEY E. YANG ${ }^{1}$, \\ RAYA S. BROWN ${ }^{1}$, JAMES C. SISSON ${ }^{1}$ and RICHARD L. WAHL ${ }^{1}$
}

${ }^{1}$ Internal Medicine, Division of Nuclear Medicine, The University of Michigan Medical Center, Ann Arbor, MI 48109, U.S.A. and ${ }^{2}$ The Hamilton Regional Cancer Center, 711 Concession St, Hamilton, Ontario, Canada L8V 1C3

(Received 26 May 1993)

\begin{abstract}
A Fast Fourier Transform method for calculating the three-dimensional dose rate distribution for murine, human-tumor xenografts is outlined. The required input includes evenly-spaced activity slices which span the tumor. Numerical values in these slices are determined by quantitative ${ }^{125} \mathrm{I}$ autoradiography. For the absorbed dose-rate calculation, we assume the activity from both ${ }^{131} \mathrm{I}-$ and ${ }^{90} \mathrm{Y}$-labeled radiopharmaceuticals would be distributed as is measured with the ${ }^{125} \mathrm{I}$ label. Two example cases are presented: an ovarian-carcinoma xenograft with an IgG $2 \mathrm{ak}$ monoclonal antibody and a neuroblastoma xenograft with meta-iodobenzylguanidine (MIBG).

Considering all the volume elements in a tumor, we show, by comparison of histograms and also relative standard deviations, that the measured ${ }^{125} \mathrm{I}$ activity and the calculated ${ }^{131} \mathrm{I}$ dose-rate distributions, are similarly non-uniform and that they are more non-uniform than the calculated ${ }^{90} \mathrm{Y}$ dose-rate distribution. However, the maximum-to-minimum ratio, another measure of non-uniformity, decreases by roughly an order of magnitude from one distribution to the next in the order given above.
\end{abstract}

\section{Introduction}

For tumor dosimetry, one would ideally have repeated, high-resolution, tomographic images so that the entire time-course of activity uptake and washout could be observed for individual voxels of interest. The three-dimensional distribution of absorbed dose up to any point could then be calculated within the tumor to high accuracy from known physical information.

Lacking such imaging both for patients and also for animal models, one can use autoradiography of excised tumors. In therapy of murine tumors, the autoradiographs can provide the activity distribution at a particular time point-the time of tumor removal. It is then possible to calculate the distribution of dose rate at that time point.

We previously published, in an abstract for the 36th Annual Meeting of the Society of Nuclear Medicine (Koral et al., 1989), preliminary results that used point dose kernels and the Fast Fourier Transform with a single autoradiograph slice to calculate the three-dimensional dose rate for murine human-tumor

*Address all correspondence and reprint requests to: Kenneth F. Koral, U of M Medical Center, 3480 Kresge III, Ann Arbor, MI 48109-0552, U.S.A. xenografts. At the meeting itself, we presented the three-dimensional dosimetry method but based on serial autoradiographs. Another group subsequently published an abstract (Roberson et al., 1990) and full paper (Roberson et al., 1992) on three-dimensional dosc calculation from serial autoradiographs with the same point dose kernels but without use of the Fast Fourier Transform. Two other groups separately derived their own point dose kernels for dosimetry calculations (Leichner et al., 1989; Lui et al., 1991).

Implantation of miniature thermoluminescent dosimeters (TLDs) in mouse tumors (Griffith et al., 1988) can add further three-dimensional information by recording the absorbed dose up to the time of sacrifice at individual spatial locations. However, drawbacks are (1) assuming that the dosimeters do not perturb the radiation field in the tumor and (2) error from inferring the absorbed dose at other spatial locations. One such previous study by Griffith et al. (1988) actually used TLD measurements in conjunction with autoradiographs. The autoradiographs showed large variations in ${ }^{131} \mathrm{I}$ activity at sacrifice for the xenograft of a Raji B-cell lymphoma in an athymic nude mouse with the labeled LYM-1 monoclonal antibody (Mab) (Griffith et al., 1988). The activity at sacrifice for a LSI74T 
colorectal-carcinoma system with B72.3 Mab appeared to be similarly non-uniform.

The B-cell-lymphoma TLD measurements corresponding to twenty volume elements gave a presacrifice absorbed dose that ranged from 392 to $1640 \mathrm{cGy}$, a maximum-over-minimum ratio of 4.2 . Fading of the TLD light output over lime was presumably a problem with these measurements. Strand et al. (1992) have shown that such fading can reduce the signal to "one third of its original value" over 9 days with the TLD in muscle tissue. [See a general review of animal TLD measurement in Yorke et al. (1993).] We are interested only in the ratio of maximum-to-minimum absorbed dose for a comparison to our computed results. As long as fading was the same fraction for both the high- and the low-dose TLD, our discussion would not be affected. The twenty regions sampled by TLD in the colorectalcarcinoma system gave a range of $330-810 \mathrm{cGy}$ which yields a somewhat smaller ratio of 2.5 .

Our purpose in this paper is to present our method, based on the Fast Fourier Transform, for three-dimensionally calculating the distribution of absorbed dose-rate at the time of sacrifice. We use two xenograft models, both different from those investigated by Griffith et al. (1988) and from that investigated by Roberson et al. (1992), as relatively independent examples. We are able to mathematically characterize the degree of non-uniformity of the different distributions over all the volume elements in a tumor as does Roberson et al. (1992). That study employs a differential dose-rate histogram; we employ three different descriptors of non-uniformity. Specifically, we compare the distribution of absorbed dose-rate with ${ }^{131} \mathrm{I}$ to the distribution of ${ }^{125} \mathrm{I}$ activity concentration because we are interested in how accurately one can infer ${ }^{131} \mathrm{I}$ dose-rate non-uniformity simply by measuring ${ }^{125} \mathrm{I}$ (or ${ }^{131} \mathrm{I}$ ) activity-concentration nonuniformity.

\section{Methods}

\section{Tumor systems}

(1) We first investigated a human ovarian-carcinoma (HTB 77 IP3) xenograft in a female nu/nu homozygous CD1 mouse. The nude mouse was injected subcutaneously with a single-cell suspension of $10 \times 10^{6}$ cells. The tumor was allowed to develop and grow over 21 days. Then, $3.7 \mathrm{MBq}(5 \mu \mathrm{g})$ of ${ }^{125}$ I-labeled 5G6.4, which is an IgG2ak murine monoclonal antibody reactive with epithelial carcinoma (Wahl et al., 1986), was injected intraperitoneally. The intraperitoneal route of administration was of interest for this system. The mouse was sacrificed 6 days later. This relatively long time after injection was chosen because we were interested in tumorspecific binding and it predominates then (Wahl et al., 1988).

(2) We then studied a human-Meyers-neuroblastoma xenograft in a female nu/nu homozygous CD1 mouse. The animals were inoculated with minced tumor as previously described (Sisson et al., 1991). When the tumor reached a diameter of approx. $1 \mathrm{~cm}$, the mouse was injected with $74 \mathrm{MBq}$ of ${ }^{125} \mathrm{I}$ metaiodobenzylguanidine (MIBG). After $6.5 \mathrm{~h}$, the animal was sacrificed and the tumor excised.

\section{Autoradiography of sample and standards}

Excised tumors were handled by quickly freezing at $-70^{\circ} \mathrm{C}$ (ovarian carcinoma) or by dipping the tissue for 45-60 $\mathrm{s}$ in iso-pentane cooled with liquid nitrogen (neuroblastoma). Frozen tissues were kept at $-70^{\circ} \mathrm{C}$. until used, then attached to the cryostat holder with OCT (Miles Scientific, Naperville, Ill.) at $-30^{\circ} \mathrm{C}$ and sectioned at -15 to $-20^{\circ} \mathrm{C}$. The frozen sections, which were $12 \mu \mathrm{m}$ thick (ovarian carcinoma) or $10 \mu \mathrm{m}$ thick (neuroblastoma), were picked up on microscope slides, with as little rotation relative to one another as possible, and air dried at room temperature. Contact autoradiography for the sections was performed by parallel positioning of these slides face down on standard sheets of Kodak XAR5 film in a cassette with an intensifying screen. For sections of ovarian carcinoma, the film was exposed for $64 \mathrm{~h}$ at room temperature; for sections of neuroblastoma, exposure was at $-70^{\circ} \mathrm{C}$ for $2 \mathrm{~h}$. The latter is a shorter procedure which we believe to be equally good.

Standards for film calibration were prepared from the livers of balb-C mice. For the ovarian xenograft, the mouse was injected with $3.7 \mathrm{MBq}$ of the ${ }^{125}$ I 5G6.4 preparation. The liver was removed, weighed, counted in a $\gamma$ counter, frozen and sectioned at the same thickness as the tumor samples. To obtain seven calibration points, a single slice was placed on each of the seven microscope slides. These were arranged on the same sheet of film as the tumor. It was assumed that the liver had a uniform concentration of activity. This assumption of uniformity was indicated by the results of the autoradiography for the individual liver slices. The total counts for the liver were converted to $\mathrm{MBq}$ by a known calibration constant in units of $\mathrm{MBq} /$ counts. The value for the activity concentration of the liver, $a$, in $\mathrm{MBq} / \mathrm{g}$ was obtained by dividing by the mass. To obtain effective values of $\mathrm{MBq} / \mathrm{g}$ for individual liver slices which were different by a factor of 2 , slice one was removed from the film after $2 \mathrm{~h}$, slice two after $4 \mathrm{~h}$, slice three after $8 \mathrm{~h}$ etc. up to slice seven after $128 \mathrm{~h}$. Since the tumor samples exposed the film for $64 \mathrm{~h}$ the individual slices of the standard had effective values of activity concentration equal to $\left(t_{i} / 64\right)^{*} a$ where $t_{i}$ are the times in hours given above $(1 \leqslant i \leqslant 7)$. This last step is sufficient because (1) the standards and samples are the same thickness and (2) film exposure is to be related to microcuries/gram. The procedure is only correct to first order because of decay but has little error $(1.5 \%$ for the worst point) because of the long half life of ${ }^{125} \mathrm{I}$ ( 60.2 days) relative to the exposure times used. For a radioisotope with a shorter half life and the same 
exposure times, it would be necessary to do the calibration with optical density plotted against cumulated activity concentration $(\mathrm{kBq} \mathrm{h} / \mathrm{mg})$ as in Jönsson et al. (1992).

For the neuroblastoma xenograft a different procedure was chosen to obtain standards of the same thickness as the tumor samples: liver sections from animals injected with $0.93,1.85,3.7,7.4,14.8$ and $22.2 \mathrm{MBq}$ of ${ }^{125} \mathrm{I}$ MIBG exposed the film being used for the tumor. This procedure required the additional assumption of similar uptake by the six mice used. Essentially, we took a new calibration because of the changed exposure conditions and also arbitrarily chose a new method for obtaining standards.

\section{Quantitative analysis of autoradiographs}

The autoradiographs were evaluated by a videodensitometric system. The light intensity passing through the film from a uniform source was evaluated and digitized $(0-255)$ as a function of position using a video camera*, lens $\dagger$ and computerł. The darkest section of film was set to have a value of 0 and the least exposed to have a value of 255 . A normalization process assured that there was equal sensitivity across the film. The digital value, $I$, for the intensity of light passing through an arbitrary pixel of the autoradiogram was then related to the highest intensity, 255 , to compute the film optical density, $D$, as follows:

$$
\log _{10} \frac{I}{255}=-D
$$

In this way, the average digital value for each standard was found and converted to an optical density.

As is customarily done, a plot of optical density versus activity concentration was made for the seven standards. The functional form of a cubic polynomial was fit to these data as shown in Fig. 1. From this form, the computed optical density of each pixel in the tumor sample was converted to a calculated activity concentration which was stored for further processing.

\section{${ }^{125}$ I data}

The original form of the ${ }^{125}$ I data for the ovarian xenograft involved 19 slices, evenly spaced every $0.6 \mathrm{~mm}$. Tumor longitudinal extent was $1.08 \mathrm{~cm}$. $\Lambda$ square $87 \times 87$ pixel array was sufficient to cover all slices; the pixel size was $244 \mu \mathrm{m}$ square. For the neuroblastoma xenograft, the original form of the data was 9 slices, evenly spaced every millimeter. Tumor longitudinal extent was $0.80 \mathrm{~cm}$. A $52 \times 68$ pixel array was used; the pixel size was $276 \mu \mathrm{m}$ square. The thickness, spacing and pixel-size differences between examples were small and mostly arbitrary. Individual slices of each tumor were assumed

*Dage-MTI New vicon.

$\dagger 25 \mathrm{~mm}, 1: 1.4$ Vidicon.

$\ddagger$ Masscomp 500 .

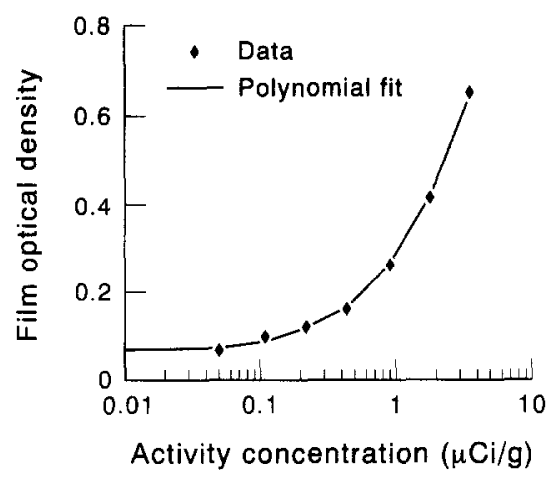

Fig. 1. Plot of average film optical density vs calculated activity-concentration for the seven standards employed for the ovarian-carcinoma xenograph. Also shown is the polynomial fit which allows the optical density for a pixel in the tumor autoradiograph to be associated with an activityconcentration.

to be properly resgistered with each other after visually locating the centroid of each slice at the same location.

\section{Three-dimensional absorbed dose-rate calculation}

A dose-rate distribution was calculated for an ${ }^{131}$ I-labeled compound by assuming that the activity distribution would be that of the ${ }^{125}$ I-labeled compound which seemed quite reasonable. A dose-rate distrubtion for ${ }^{90} \mathrm{Y}$ was calculated by making the same assumption, although labeling MIGB with ${ }^{90} \mathrm{Y}$ was unlikely. The results in this case are mainly a second demonstration of the effects of the longerrange $\beta$-particle of ${ }^{90} \mathrm{Y}$.

The two sets of multiple-slice ${ }^{125}$ I activity-concentration data, one for each tumor system, were analyzed using a program, TDRD, on a Digital VAX 8300 computer. The program calculated the threedimensional dose-rate distribution, $D(x, y, z)$, in a homogencous water-equivalent medium resulting from the specified activity-concentration distribution $A(x, y, z)$ using a Fast Fourier Transform approach. Applications of an earlier version of TDRD in the calculation of $D(x, y, z)$ for radially-symmetrical activity-concentration distributions have been described previously (Kwok et al., 1985). In the earlier version, Loevinger's $\beta$-point-source dose function (Loevinger et al., 1956) was used, whilc in the present version of TDRD the $\beta$-dose point kernels derived from Monte Carlo calculations of electron transport in water were incorporated (Prestwich et al., 1989) to increase accuracy.

To input a non-symmetrical $A(x, y, z)$ into the program, the distribution had to be digitized into evenly-spaced $64 \times 64$-pixel activity-concentration slices, $A_{i}(x, y), 1 \leqslant i \leqslant 32$. The number of slices had to be a power of 2 . Consequently, the 19 slices of the ovarian xenograft activity-concentration data were interpolated longitudinally to 32 evenly-spaced activity-concentration slices. The original $87 \times 87$ matrix size for each slice was reduced in resolution in 
both $x$ and $y$ directions by summing every four neighboring pixels into one. For the neuroblastoma xenograft, the original 9 slices were interpolated longitudinally to give 16 evenly-spaced $A_{i}(x, y)$. The original $52 \times 68$ matrix size for each $A_{i}(x, y)$ was reduced in resolution by a factor of two in the second direction. The three-dimensional Fourier transform of the reformatted activity-concentration data was then evaluated together with the three-dimensional Fourier transform of the dose point kernel of the radionuclide, either ${ }^{90} \mathrm{Y}$ or ${ }^{131} \mathrm{I}$. After multiplying the two Fourier transforms and evaluating the inverse Fourier transform of the product, $D(x, y, z)$ was calculated. For each of the two radionuclides, $D(x, y, z)$ was output into a disk file slice by slice in both graphical and numerical form.
A flow chart of the general program appears in Fig. 2. Note that a subroutine USRAD, can still be used to generate hypothetical, radially-symmetric, activity distributions. This provision was not employed in this paper. Also note that if the activity distribution is digitized to a resolution that is greater than $32 \times 32$, then a subroutine, SPLIT, is called to divide the activity-concentration matrix into submatrices and appropriate steps are followed.

It should be pointed out that photon doses from ${ }^{131}$ I were not included because they would contribute less than several percent of the beta doses for tumor volumes less than a few centimeters in diameter. The accuracy of TDRD has been checked indirectly against phantom studies (Wessels et al., 1986) and directly against other computational methods

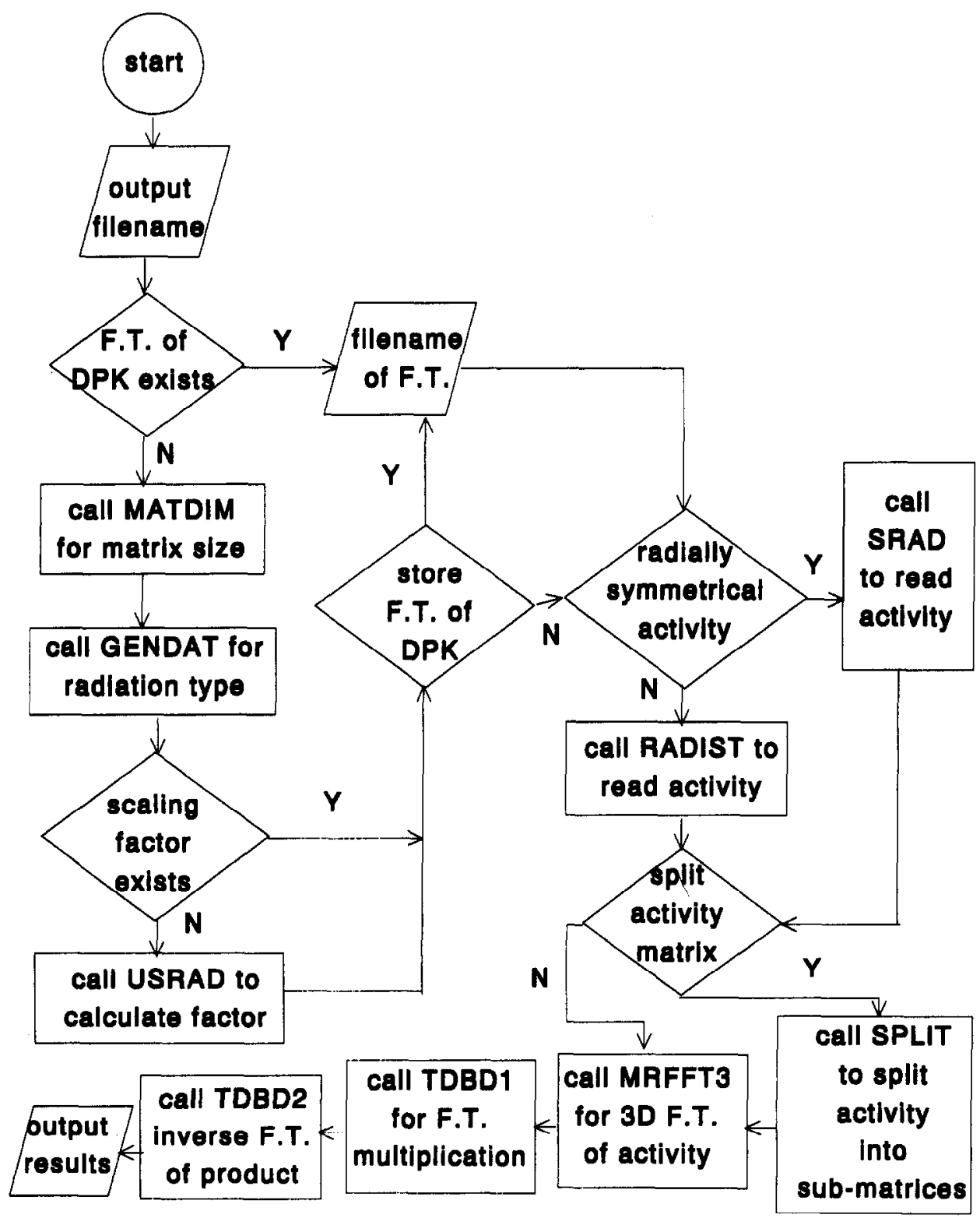

Fig. 2. Flow chart of three-dimensional absorbed dose-rate program. Note that F.T. refers to the Fast Fourier Transform and DPK stands for dose point kernel. 

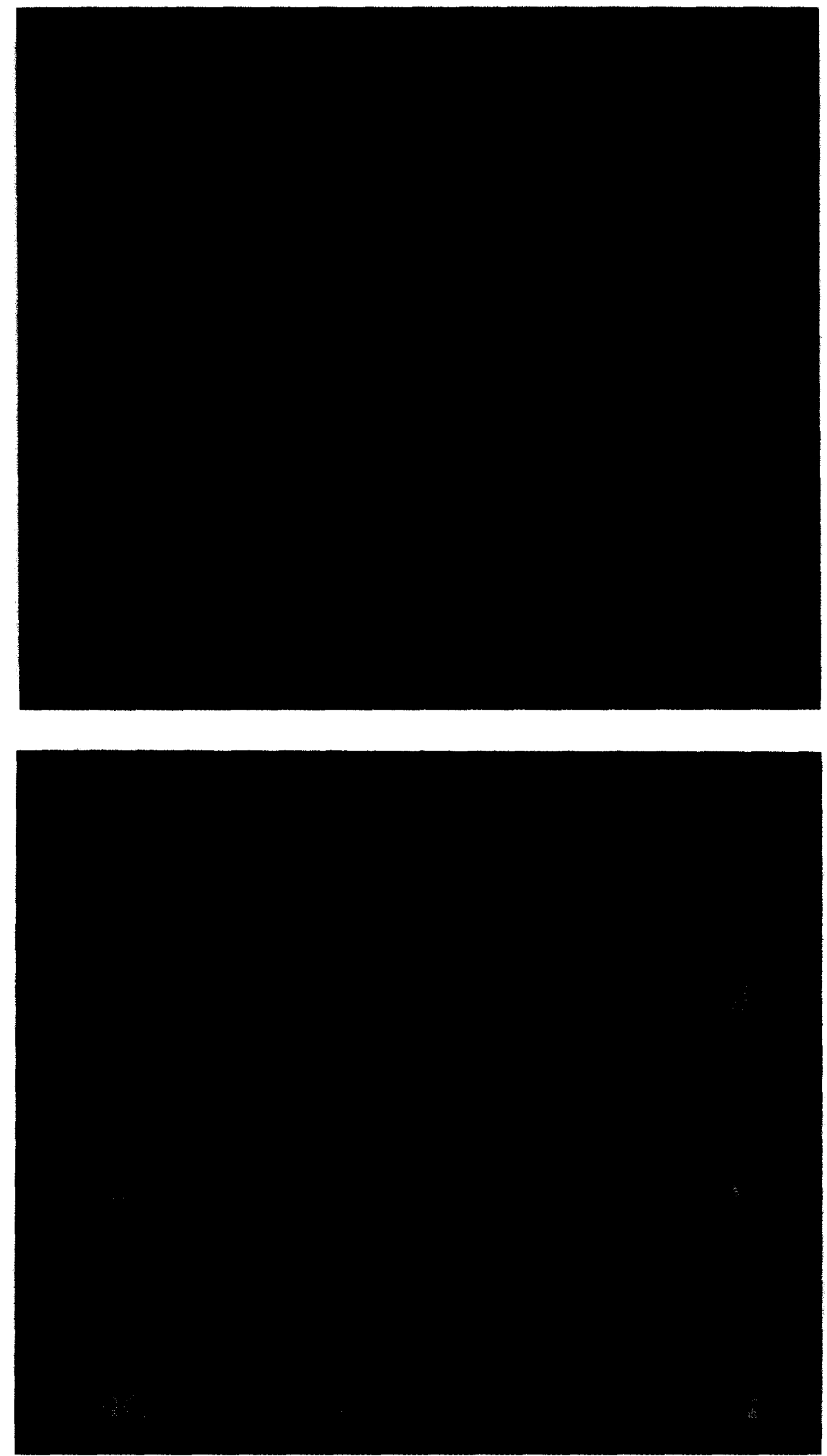

Figs 3 (top) and 4 (bottom). Captions on p. 906. 


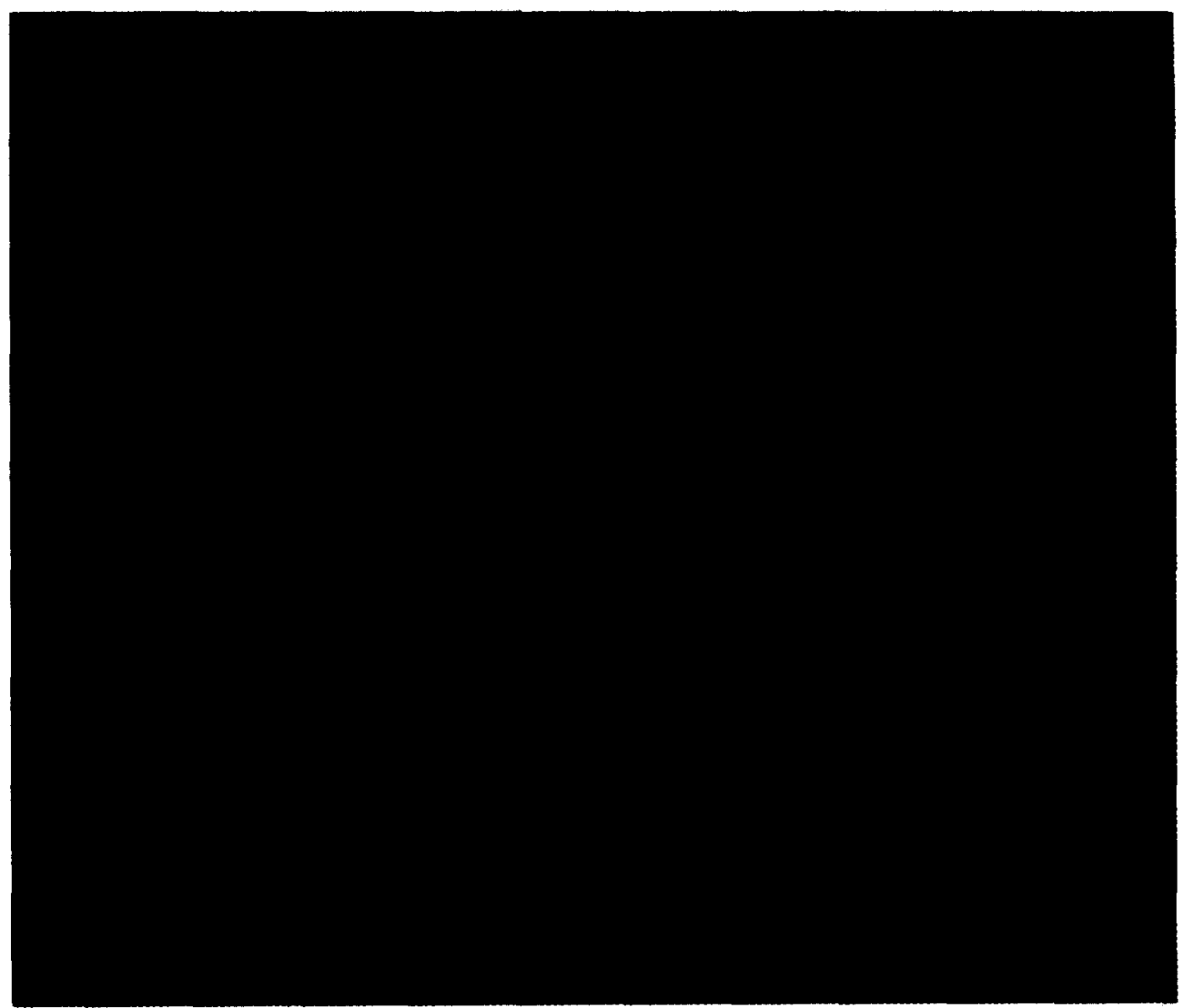

Fig. 5. Comparison of three distributions for the 18th plane of the ovarian-carcinoma xenograft shown in Fig. 3. The activity distribution measured with ${ }^{125} \mathrm{I}$ and the dose-rate distribution calculated for the $\beta$-emissions from ${ }^{131} I$ are almost equally non-uniform. The non-uniformity is reduced assuming labeling with ${ }^{90} \mathrm{Y}$. The color scale is independently related to the values in the particular distribution.

(Werner et al., 1988). In the first study, TLD fading may have distorted the results.

\section{Characterization of distributions}

We characterized a multi-plane distribution in three ways: (1) by the ratio of the largest value divided by the smallest, (2) by a histogram of values and (3) by a single statistic representing non-uniformity. The determination of the tumor edge was done by including all non-zero values in the activity-concentration distribution. All voxels within the edge were then checked to find the largest and smallest values and the ratio of these values was computed. Also the average value over all voxels was found. To calculate a histogram, bar width was set at 0.2 of the average value. Then, the number of voxels within each range $(0-0.2$ times average, $0.2-0.4$ times average etc) was found. Values for bars which were beyond 2.0 times average were not plotted for convenience. A very uniform distribution would be represented by two bars of equal height at 0.8-1.0 and 1.0-1.2.

For the single statistic, we chose relative standard deviation (also called coefficient of variation), that is, the standard deviation divided by the mean. This value is directly proportional to non-uniformity and has a smallest possible value of zero. That is, if all voxels had exactly the same value (no non-uniformity), the standard deviation and, thus, the relative standard deviation would be zero. The maximum value was finite but unlimited.

\section{Results}

The 32-plane result of longitudinal interpolation for the human-ovarian-cancer xenograft is shown in Fig. 3. It is seen that the region of highest activity concentration changes location as one advances from plane to plane. No single slice has uniform activity

Fig. 3 (p. 905, top). Distribution of activity-concentration for the ovarian-cancer xenograft after interpolation. Spacing is $348 \mu \mathrm{m}$. The color scale is related to the activity-concentration of the slices as a group. That is, a given color represents the same activity-concentration in each slice. The slice sequence is left to right, top to bottom.

Fig. 4 (p. 905, bottom). Distribution of activity-concentration for the neuroblastoma xenograft after interpolation. Spacing is $530 \mu \mathrm{m}$. Color scale and slice sequence as in Fig. 3 . 
concentration. The ratio of maximum pixel value over minimum pixel value is given in Table 1.

The 16-plane result of interpolation for the neuroblastoma-tumor xenograft is shown in Fig. 4. Here some of the individual slices appear fairly uniform in their activity concentration, although the level can change sharply over only a one-slice difference. The maximum to minimum ratio is given in Table 1.

For the plane approximately mid-way from the ends of the ovarian-carcinoma tumor (plane number 18 out of 32), Fig. 5 shows the ${ }^{125}$ I activity-concentration distribution as well as the calculated ${ }^{131} \mathrm{I}$ dose-rate and ${ }^{90} \mathrm{Y}$ dose-rate distributions. The ${ }^{125} \mathrm{I}$ activity-concentration distribution appears to be only slightly more non-uniform than the ${ }^{131}$ I dose-rate. The ${ }^{90} \mathrm{Y}$ dose-rate appears to be the least nonuniform.

A histogram comparison of the same three distributions, but for the entire tumor rather than a single plane, is given in Fig. 6. There are more values at the lowest extreme for the activity-concentration than for either dose-rate distribution and also more with ${ }^{131} \mathrm{I}$ than with ${ }^{90} \mathrm{Y}$.

As given in Table 1, there is a maximum-tominimum ratio of 120 for the ${ }^{131} \mathrm{I}$ dose-rate distribution, and one of 15.4 for the ${ }^{90} \mathrm{Y}$ dose-rate distribution. The change is roughly an order of magnitude as is the change between ${ }^{125}$ I activity and ${ }^{131}$ I dose-rate.

For the neuroblastoma xenograft (not shown) there are again more values at the lowest extreme for the activity-concentration than for either dose-rate distribution and also more with ${ }^{131} \mathrm{I}$ than with ${ }^{90} \mathrm{Y}$. The number of voxels between 0 and 0.2 of the mean are $9.7,6.5$ and $0.0 \%$ of all voxels, respectively. For the activity-concentration and for absorbed dose-rate with ${ }^{131} \mathrm{I}$, the distribution is skewed: the highest bars are for the ranges slightly greater than the mean. For absorbed dose-rate with ${ }^{90} \mathrm{Y}$, the distribution is more symmetric. The maximum-to-minimum dose-rate ratios are given in Table 1.

Relative standard deviation is given in Table 2. For the ${ }^{125} \mathrm{I}$ activity distribution, it is $65.7 \%$ for the ovarian-carcinoma tumor and $48.0 \%$ for the neuroblastoma tumor. (The ovarian activity is thus more non-uniform.) The non-uniformity decreases as one changes from activity-concentration to ${ }^{131} \mathrm{I}$ doserate and finally to ${ }^{90} \mathrm{Y}$-dose-rate. The same trend appeared in the single ovarian-carcinoma slice in Fig. 5.

Table 1. Ratio of maximum pixel value over minimum pixel value for specified distribution in ovarian and neuroblastoma xenografts

\begin{tabular}{lcc}
\hline & \multicolumn{2}{c}{ Ratio of Maximum-over-minimum } \\
\cline { 2 - 3 } Distribution & Ovarian & Neuroblastoma \\
\hline${ }^{125}$ I activity-concentration & 1140 & 3540 \\
${ }^{131}$ I absorbed dose-rate & 120 & 188 \\
${ }^{90} \mathrm{Y}$ absorbed dose-rate & 15.4 & 8.15 \\
\hline
\end{tabular}

\section{Discussion}

We have found the activity-concentration distribution of two tumor-xenograft systems, ovarian carcinoma and neuroblastoma, to be non-uniform. Such was also the case for the normal rat kidney using an ${ }^{11}$ In-labeled anti $\left.\mathrm{CEA}-\mathrm{F}(\mathrm{ab})_{2}\right)_{2}$ monoclonal antibody (Jönsson et al., 1992). It was also the case for the tumor systems investigated by Griffith et al. (1988) for lymphoma and colorectal carcinoma. However, early after injection, our neuroblastoma results do not show activity predominantly at the tumor surface
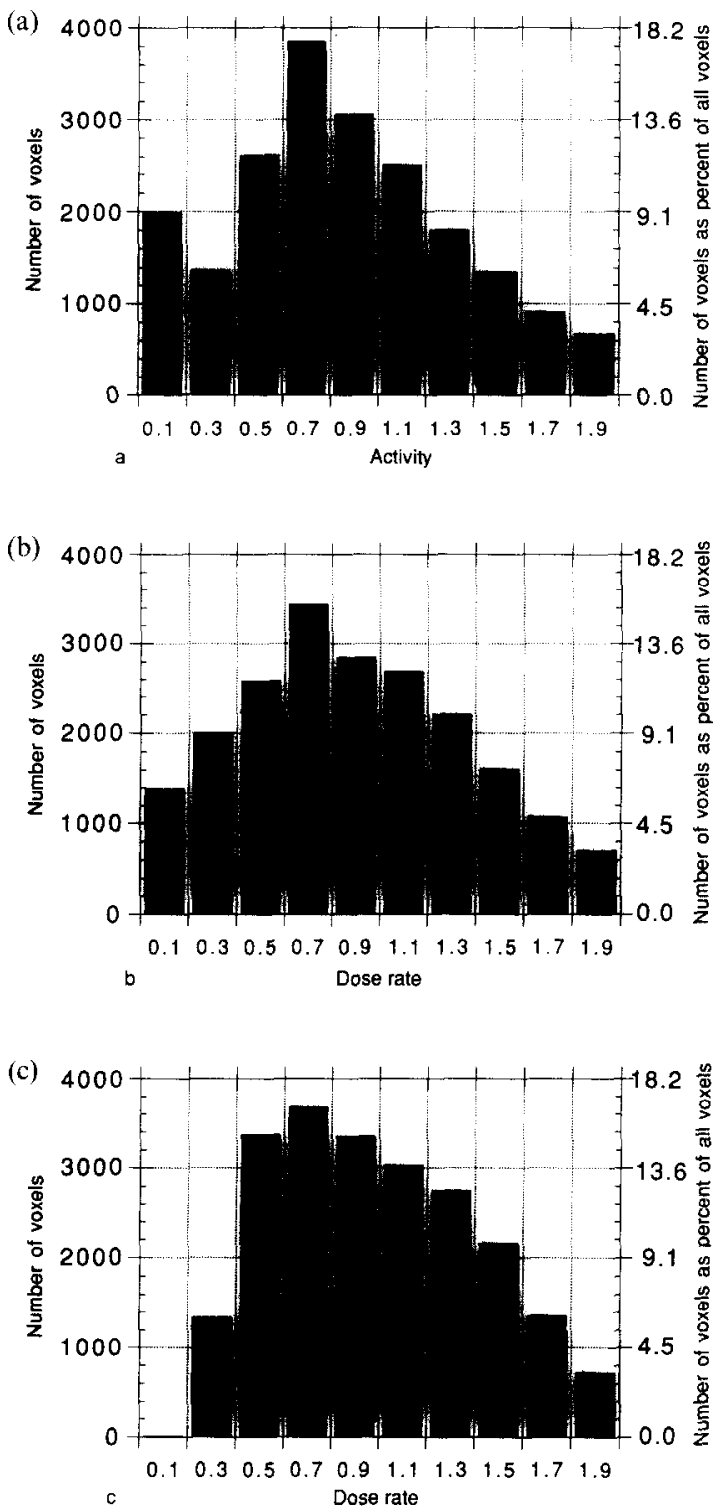

Fig. 6. Histogram comparison of three distributions over all the volume elements in the ovarian-carcinoma xenograft: the ${ }^{125}$ I activity-concentration (a), the ${ }^{131} \mathrm{I}$ dose-rate distribution (b), and the ${ }^{90} \mathrm{Y}$ dose-rate distribution (c). The number of voxels within a range is plotted against the midpoint of the range. Each midpoint is a fraction of the mean value for the entire tumor. In (a), (b) and (c) respectively, 1856,1466 and 243 voxels lie beyond twice the average and are not plotted. 
as was the case with LS147T human-colon-cancer xenografts with ${ }^{131}$ I-labeled 17-1A Mab (Roberson et al., 1992).

We have demonstrated that the dose-rate distribution with ${ }^{131} I$ is similar to that for the ${ }^{125} I$ activityconcentration except as characterized by the maximum-to-minimum ratio. This ratio reflects the range of the distribution. It has practical importance, for example, when one is concerned that all voxels receive at least some lower limit of radiation absorbed dose. However, since the ratio is determined by the values in only two voxels, error in these values can distort its value. In this respect, one could, perhaps, derive more use from an average-to-minimum ratio since it sould be more error-resistant. The general similarity in the two distributions, excluding the maximum-to-minimum ratio, is not surprising given that the range of the ${ }^{131} I \beta$-particle and the imaging resolution are similar.

In the case of the ovarian-carcinoma xenograft, results for a middle plane in the tumor show the ${ }^{90} \mathrm{Y}$ dose-rate to be the least non-uniform. This result is cxpected given the relatively long range of the ${ }^{90} \mathrm{Y}$ $\beta$-particle. It also is consistent with the calculated results for a $2 \times 2 \mathrm{~mm}$ region in a Raji B-cell tumor: Fig. $11\left({ }^{131} \mathrm{I}\right)$, compared to Fig. $12\left({ }^{90} \mathrm{Y}\right)$ in Griffith et al. (1988). In their calculation, however, the results at the edges may be incorrectly low because the activity outside the region was neglected. Moreover, a measured, three-dimensional activity distribution was not used; rather, the measured activity of the plane of interest was simply replicated above and below that plane.

For the dose-rate distribution from ${ }^{131} I$ at the time of sacrifice, the maximum-over-minimum ratio is 188 for the ovarian carcinoma system and 120 for the neuroblastoma xenograft. These values are much larger than the pre-sacrifice absorbed-dose maximum-to-minimum ratios calculated from the TLD samples published by Griffith et al. (1988). Recall their observed ratios of 4.2 for B-cell lymphoma and 2.5 for colorectal carcinoma. We sampled 21,994 and 9698 voxels in the ovarian and neuroblastoma xenografts, respectively, however, while they sampled only 20 locations in each of their systems. The much greater number of samples makes finding a much larger maximum-to-minimum ratio more likely, if it physically exists.

There is one methodological point to discuss: the question of whether the three-dimensional registration was accurate. A procedure for registering

Table 2. Relative standard deviation* for specified distributions in ovarian and neuroblastoma xenografts

\begin{tabular}{lcc}
\hline & \multicolumn{2}{c}{ Relative standard deviation } \\
\cline { 2 - 3 } Distribution & Ovarian & Neuroblastoma \\
\hline${ }^{125}$ I activity-concentration & $65.7 \%$ & $48.0 \%$ \\
${ }^{131} \mathrm{I}$ absorbed dose-rate & 60.1 & 45.6 \\
${ }^{90} \mathrm{Y}$ absorbed dose-rate & 43.5 & 38.5 \\
\hline
\end{tabular}

*A greater value implies more non-uniformity. both the horizontal and vertical centroids of all the slices could easily be automated. However, this would not necessarily yield the true threedimensional orientation. Obtaining that orientation would probably require two marker "wires" which could be inserted through the tumor without distorting it but yet be cut by the microtome. Even then, one cannot be sure of retaining the in vivo shape of the tumor, so the problem remains, to some extent, unless one achieves high-resolution in vivo imaging.

We note that our three-dimensional dose-rate computer program appears faster than that of reference (Roberson et al., 1992) because we use the Fast Fourier Transform. They report that calculation of a three-dimensional dose-rate distribution on a grid of $200 \mu \mathrm{m}$ takes approx. $100 \mathrm{~h}$ on a VAX $8800 \mathrm{com}$ puter whereas we calculated both the ${ }^{131} \mathrm{I}$ and the ${ }^{90} \mathrm{Y}$ dose-rate distributions on a grid of $550 \mu \mathrm{m}$ in $30 \mathrm{~min}$ on a VAX 8300 . Their finer grid accounts for some, but not all, of the time difference. They acknowledge that use of the Fast Fourier Transform would reduce their calculation time.

Also, our program can produce absolute values. This fact is fairly clear as an extension of the results of Kwok et al. (1985). We have not presented nor made explicit use of that capability here.

Lastly, the problem of obtaining all the dosimetry information from a tumor which has taken up radioactivity still remains, as we mentioned in the Introduction. It will probably wait on the development of new, high-resolution, small-animal tomographs (see Rogers et al., 1993; Green et al., 1992) as does solution of the registration problem. Other comments on the general dosimetric problem can be found in a recent editorial by Roberson (1992).

\section{Summary}

As detailed in the text, our absorbed dose-rate calculations are based on the assumption that, with ${ }^{131}$ I labeling as well as ${ }^{90} \mathrm{Y}$ labeling, the activity distribution will be the same as with ${ }^{125} \mathrm{I}$ labeling. Within that assumption, our results show: (1) that with ${ }^{131} \mathrm{I}$, the general non-uniformity of the activity distribution is similar to the general non-uniformity of the dose-rate distribution; (2) that the ${ }^{90} \mathrm{Y}$ dose-rate distribution is generally more uniform than either the activity distribution or the ${ }^{131} \mathrm{I}$ dose-rate distribution; and (3) that the ratio for the maximumvalued voxel over the minimum-valued voxel decreases by roughly an order of magnitude between the activity-concentration distribution and the ${ }^{131} I$ dose-rate distribution and then again between the ${ }^{131} \mathrm{I}$ and the ${ }^{90} \mathrm{Y}$ dose-rate distributions. The first two results are new because they hold for distributions over all the volume elements in a tumor. They extend similar previous findings for single slices through a tumor. 
Acknowledgements - The authors acknowledge the autoradiograph-analysis help of Will Jaynes, the computer assistance of Steven Buchbinder, Xiaohan Wang and Jian-qiao Luo and the animal work of Anaira Clavo and Gayle A. Jackson. The secretarial assistance of Patricia Haines is also recognized. This publication was supported by PHS Grant Nos ROI CA38790, CA50872 and PO CA42768 awarded by the National Cancer Institute. Its contents are solely the responsibility of the authors and do not necessarily represent the official views of the National Cancer Institute.

\section{References}

Green M. V., Markowitz A., Tedder T. E., Andrich M. P., Owens E. S. and Neumann R. D. (1992) SPECT intaging in small animals. J. Nucl. Med. 33, 852 (abstract).

Griffith M. H., Yorke E. D., Wessels B. W., DeNardo G. L. and Neacy W. P. (1988) Direct dose confirmation of quantitative autoradiography with micro-TLD measurements for radioimmunotherapy. J. Nucl. Med. 29, 1795-1809.

Jönsson B.-A., Strand S.-E. and Larsson B. S. (1992) A quantitative autoradiographic study of the heterogeneous activity distribution of different indium-111-labeled radiopharmaceuticals in rat tissues. J. Nucl. $\mathrm{Med}$. 33, 1825-1833.

Koral K. F., Kwok C., Yang F. E. and Wahl R. L. (1989) Variations in absorbed dose for radiolabeled monoclonal antibodies in a murine human-tumor xenograft. $J . \mathrm{Nucl}$. Med. 30, 777 (abstract).

Kwok C. S., Prestwich W. V. and Wilson B. C. (1985) Calculation of radiation doses for nonuniformly distributed $\beta$ and $\gamma$ radionuclides in soft tissue. Med. Phys. 12, 405-412.

Leichner P. K., Hawkins W. G. and Yang N.-C. (1989) A generalized, empirical point-source function for beta-particle dosimetry. Antibody Immunoconj. Radiopharm. 2, 125-144.

Loevinger R., Japha E. M. and Brownell G. L. (1956) Radiation Dosimetry (Edited by Hine G. J. and Brownell G. L.), pp. 693-799. Academic Press, New York.

Lui J. C., Bolch W. E. and Poston J. W. Sr (1991) Calculations of scaled dose kernels for electrons in tissue. In Proceedings of the 5th International Radiopharmaceutical Dosimetry Symposium, Oak Ridge Associated Universities, Oak Ridge, Tenn., 7-10 May 1991.

Prestwich W. V., Nunes J. and Kwok C. S. (1989) Beta dose point kernels for radionuclides of potential use in radioimmunotherapy. $J$. Nucl. Med. 30, 1036-1046 and 1739 for correction.

Roberson P. L., Buchsbaum D. J., Heidorn D. B. and Ten Haken R. K. (1990) Three-dimensional tumor dosimetry for I-131 labeled Mo $\mathrm{Ab}$ using serial autoradiography. Antibody Immunoconj. Radiopharm. 3, 78 (abstract).

Roberson P. L., Buchsbaum D. J., Heidorn D. B. and Ten Haken R. K. (1992) Three-dimensional tumor dosimetry for radioimmunotherapy using serial autoradiography. Int. J. Radiat. Oncol. Biol. Phys. 24, 329-334.

Roberson P. L. (1992) Quantitative autoradiography for the study of radiopharmaceutical uptake and dose heterogeneity. J. Nucl. Med. 33, 1833-1834 (Editorial).

Rogers W. L., Slosar J., Hua L., Chiao P., Zhang Y. and Clinthorne N. H. (1993) A high resolution slit aperture for imaging small animals with SPECT. $J$. Nucl. Med. 34, 9P (abstract).

Sisson J. C., Brown R., Zasadny K., Normolle D. P., Fisher S., Moon S., Gutowski T. and Wahl R. L. (1993) Biodistribution and therapeutic effects of radiolabeled metaiodobenzylguanidine in a human neuroblastoma xenograft. Antibody Immunoconj. Radiopharm. 5, 23-26.

Strand S.-E., Svedberg A., Strandh M. and Norrgren K. (1992) Parameters affecting the accuracy of in vivo dosimetry with mini-TLD. Med. Phys. 19, 781 (abstract).

Wahl R. L., Barrett J., Geatti O., Leibert M., Wilson B. S. Fisher S. and Wagner J. G. (1988) The intraperitoneal delivery of radiolabeled monoclonal antibodies: studics on the regional delivery advantage. Cancer Immunol. Immunother. 26, 187-201.

Wahl R. L., Liebert M., Biesman B., Roberts J., Jackson G., Kronberg S. and Laino L. (1986) Production and characterization of a murine monoclonal antibody reactive with ovarian and other epithelial carcinomas. In Proceedings of the $A A C R$ 27, 355

Werner B. L., Kwok C. S. and Das I. J. (1988) Dose distributions in regions containing beta sources: large spherical source regions in a homogeneous medium. $\mathrm{Med}$. Phys. 15, 358-363.

Wessels B. W. and Griffith M. H. (1986) Miniature thermoluminescent dosimeter absorbed dose measurements in tumor phantom models. J. Nucl. Med. 27 1308-1314.

Yorke E. D., Williams L. E., Demidecki A. J., Heidorn D. B., Roberson P. L. and Wessels B. W. (1993) Multicellular dosimetry for beta-emitting radionuclides: autoradiography, thermoluminescent dosimetry and three-dimensional dose calculations. Med. Phys. 20, Pt2,543-550. 